\title{
Choriocarcinome Métastatique Diagnostiqué Et Pris En Charge Aux Urgences Dans Un Pays À Faible Ressources
}

\author{
Naharisoa Giannie Rasamimanana, \\ Service Des Urgences Et Soins Intensifs (Susi), Chu Pzaga Mahajanga \\ Jean José Christien Rajaonarison, \\ Complexe Mère-Enfant, Chu Pzaga Mahajanga \\ Dolly Velonjara Tohaina, \\ Accueil Triage Urgence Chu Mahavoky Atsimo Mahajanga \\ Valérie Refeno, \\ Service D'oncologie Médicale, Chupzaga Mahajanga \\ Nasolotsiry Enintsoa Raveloson,
}

Accueil Triage Urgence Et Réanimation Chujr Befelatanana Antananarivo

Pierana Gabriel Randaoharison,

Complexe Mère-Enfant, Chu Pzaga Mahajanga

Doi:10.19044/esj.2020.v16n12p385 URL:http://dx.doi.org/10.19044/esj.2020.v16n12p385

\section{Résumé}

Le choriocarcinome est une tumeur rare à fort potentiel métastatique pouvant résulter de la dégénérescence d'une môle hydatiforme. Vu au stade de métastase pulmonaire, un tableau de détresse respiratoire inaugural oriente le malade aux urgences. Nous rapportons le cas d'une patiente âgée de 31 ans vivant en milieu rural dans un pays à faibles ressources, évacuée en urgence dans le centre hospitalier de référence pour une détresse respiratoire simulant une pneumopathie aigue grave. Elle présente une anémie sévère à $23 \mathrm{~g} / \mathrm{L}$ d'hémoglobine et un saignement vaginal minime. Les examens paracliniques faisaient découvrir des métastases pulmonaire et hépatique d'un choriocarcinome gestationnel. Le retard de diagnostic associé à une absence de suivi régulier clinique, biologique et échographique met en jeu le pronostic vital d'une telle patiente. Une prise en charge adéquate respiratoire et hémodynamique en soins intensifs associée à une polychimiothérapie et une éventuelle chirurgie améliorerait le pronostic de la patiente.

Mots clés : Détresse respiratoire, Urgences, môle hydatiforme, choriocarcinome, métastases 


\title{
Metastatic Choriocarcinoma Diagnosed and Treated in an Emergency Department in a Low-Income Country
}

\author{
Naharisoa Giannie Rasamimanana, \\ Service Des Urgences Et Soins Intensifs (Susi), Chu Pzaga Mahajanga \\ Jean José Christien Rajaonarison, \\ Complexe Mère-Enfant, Chu Pzaga Mahajanga \\ Dolly Velonjara Tohaina, \\ Accueil Triage Urgence Chu Mahavoky Atsimo Mahajanga
}

Valérie Refeno,

Service D'oncologie Médicale, Chupzaga Mahajanga

Nasolotsiry Enintsoa Raveloson,

Accueil Triage Urgence Et Réanimation Chujr Befelatanana Antananarivo

Pierana Gabriel Randaoharison,

Complexe Mère-Enfant, Chu Pzaga Mahajanga

\begin{abstract}
Choriocarcinoma is a rare tumour with high metastatic potential that can result from the degeneration of a hydatiform mole. Seen at the stage of pulmonary metastasis, an inaugural respiratory distress chart directs the patient to the emergency room. We report the case of a 31-year-old patient living in rural areas in a low-resource country, rushed to the referral hospital for respiratory distress simulating severe acute lung disease. She has severe anaemia at $23 \mathrm{~g} / \mathrm{L}$ of hemoglobin and minimal vaginal bleeding. Paraclinical examinations revealed pulmonary and liver metastases of a gestational choriocarcinoma. Delayed diagnosis associated with a lack of regular clinical, biological and ultrasound follow-up involves the life-threatening prognosis of such a patient. An adequate respiratory and hemodynamic management in intensive care combined with polychemotherapy and possible surgery would improve the patient's prognosis.
\end{abstract}

Keywords: Respiratory Distress, Emergency, Hydatidiform Mole, Choriocarcinoma, Metastases 


\section{Introduction}

Le choriocarcinome est une tumeur maligne rare ayant un fort potentiel métastatique (Golfier 2010). Non diagnostiqué précocement et sans traitement, le pronostic est fatal. Il s'intègre dans les maladies trophoblastiques gestationnelles. L'objectif de ce travail est de rapporter un cas de choriocarcinome métastatique de diagnostic difficile et de discuter de la prise en charge dans un service d'urgence où les ressources sont insuffisantes.

\section{Observation}

Il s'agit d'une femme de 31 ans, référée du centre hospitalier de District de Mampikony pour une dyspnée et une pneumopathie. Ce centre se trouve dans une zone rurale à $250 \mathrm{~km}$ du centre hospitalier Universitaire PZAGA de Mahajanga, Madagascar. Arrivée aux urgences, elle présentait une détresse respiratoire avec une tachypnée à 33/mn, un tirage, un battement des ailes du nez, une respiration abdominale associée à une toux sèche et une hémoptysie. La saturation d'oxygène à l'air ambiant était à $89 \%$ associée à une cyanose des lèvres et des extrémités, une tachycardie, une hypotension artérielle à 80/60mmhg. Elle présentait une anémie sévère à $23 \mathrm{~g} / \mathrm{L}$, avec signes de mauvaise tolérance à l'anémie et à l'hypoxie tels qu'une dyspnée même au repos, une obnubilation et une agitation. Elle était asthénique, amaigrie, et non fébrile. La leucocytose était normale à 4900/mm3 et la thrombopénie à $90000 / \mathrm{mm} 3$. Le trouble de conscience rendait l'interrogatoire difficile et les renseignements cliniques très pauvres au début. La femme se plaignait depuis environ trois mois de toux chronique associée à une fièvre vespérale constituant un motif fréquent de consultation en milieu rural. Elle a été traitée pour une broncho-pneumopathie avec des expectorants et des antibiotiques à visée respiratoire sans aucune amélioration. En outre, la femme avait eu trois grossesses antérieures sans particularité avec deux enfants vivants, le dernier étant âgé de 5 ans. La consultation de son carnet de santé nous a fait découvrir qu'elle a subi une aspiration manuelle intra-utérine pour un avortement spontané avec rétention de produits de conception quatre mois avant son admission aux urgences. Elle n'a pas reçu d'information concernant la suite de l'aspiration. Il persistait une métrorragie minime chronique depuis ces quatre derniers mois. Elle n'avait bénéficié ni de traitement spécifique ni de dosage de l'hormone Gonadotrophine chorionique humaine (Béta-hCG) ni d'autre explorations malgré la persistance du saignement après cette intervention. L'auscultation pulmonaire a retrouvé des râles crépitants diffuses dans les deux champs pulmonaires. Il n'y avait pas de signes d'insuffisance cardiaque. L'examen gynécologique découvrait un utérus augmenté de volume, à mi-distance entre le pubis et l'ombilic, dur, indolore, peu mobilisable et un saignement d'origine endo-utérin. Le col était d'aspect normal. L'examen cyto-bactériologique du crachat et la recherche de BAAR 
étaient revenus négatifs. Le dosage qualitatif de béta hCG dans les urines était positif car le $\mathrm{CHU}$ ne disposait pas de test quantitatif. La radiographie pulmonaire montrait de multiples opacités parenchymateuses bilatérales (Fig.1). L'échographie abdomino-pelvienne révélait la présence d'une image en nid d'abeille intra-utérine mesurant 63,8 x 57,2mm (Fig.2), un foie nodulaire à bords irréguliers en faveur d'une métastase (Fig.3), un épanchement pleural et intra abdominal. Le diagnostic de choriocarcinome avec métastases pulmonaire et hépatique a été retenu (Stade IV de FIGO, classé tumeur à haut risque avec un score pronostic de l'OMS à 9). L'état hémodynamique s'était amélioré après une transfusion sanguine de deux culots globulaires avec un plasma frais congelé et une prise en charge des urgences vitales. Malgré la mise en place d'une oxygénothérapie au masque à haute concentration, les paramètres respiratoires n'étaient pas satisfaisants. Une prise en charge en oncologie a été immédiatement débutée. Toutefois la patiente est sortie contre avis médicale malgré une information sur le stade de sa maladie et les principes de traitement.

\section{Discussion}

La môle hydatiforme concerne une à trois pour mille grossesses. Le risque de tumeur trophoblastique est de 10 à $20 \%$ après une môle hydatiforme complète et de l'ordre de $0,5 \%$ après une môle hydatiforme partielle (Golfier, 2010). Une étude malgache à l'Hôpital Universitaire de Gynécologie Obstétrique de Befelatanana a retrouvé deux cas sur 46 grossesses môlaires sur une période de un an (Rajaonarison, 2015).

Bien que rare cette pathologie peut mettre en jeu la vie de la femme lorsqu'elle est vue tardivement. Le choriocarcinome peut être révélé par une métastase et par ordre décroissant les localisations pulmonaires sont majoritaires soit $80 \%$, le vagin (30\%), le pelvis $(20 \%)$, le cerveau $(20 \%)$ et le foie (10\%) (Golfier, 2010). Les symptômes constatés aux urgences étaient trompeurs au début vu la prédominance des signes respiratoires et la prise en charge antérieure d'une pneumopathie. En effet, l'aspect clinique et radiologique peut simuler une pneumonie semblablement au cas décrit par Mirambo et al. où le diagnostic de choriocarcinome n'a pu être détecté que très tardivement huit mois après les premiers symptômes respiratoires (Mirambo, 2010). Olezac et al. ont décrit une forme clinique plus discrète de métastase pulmonaire d'un choriocarcinome dont le signe d'appel était une toux persistante (Olezac, 2009). Sierra Bergua et al. ont noté la survenue de symptômes respiratoires à type d'hémoptysie associée à une fièvre, huit mois après 1'accouchement (Sierra Bergua, 2008) et pour El Fekih et al. ils apparaissent deux mois après un accouchement d'un bébé mort-né (El Fekih, 2010). Ainsi, les symptômes respiratoires sont hétérogènes et non spécifiques (Mirambo, 2010). L'invasion pulmonaire du choriocarcinome se fait par 
diffusion hématogène d'un micro embole métastatique se manifestant par un œdème pulmonaire, un Syndrome de détresse respiratoire (SDRA) ou une hypertension artérielle pulmonaire (Olezac, 2009) (Mehrishi, 2004). Il a été évoqué que ce sont surtout les métastases à localisations cérébrale et hépatique du choriocarcinome qui mettent en jeu le pronostic vital du patient du fait des hémorragies massives intracérébrales et de la résistance au traitement (Golfier, 2010). Cependant, la prise en charge initiale de la détresse respiratoire aux urgences d'une métastase pulmonaire reste difficile en milieu défavorisé où les plateaux techniques sont insuffisants. L'hypoxémie et l'inondation alvéolaire par l'hémoptysie représentent la source d'aggravation de l'état de la patiente. Il est connu que cette pathologie est potentiellement curable lorsque une polychimiothérapie est démarrée de façon adéquate (Golfier, 2010 Olezac, 2009 Chen, 2017). La ventilation invasive est indiquée devant un SDRA, toutefois il a été évoquée que cette technique constitue une source de décès fréquent en réanimation par complication infectieuse ou de barotraumatisme chez le patient cancéreux et fragile (Cuvelier, 2003), de plus le coût de la sédation et de la respiration artificielle n'est pas à la portée des patients. La ventilation non invasive pourrait être proposée en premier lieu vue l'échec de l'oxygénothérapie par le masque à haute concentration chez cette patiente. Son intérêt a été démontré chez le patient cancéreux hypoxémique (Cuvelier, 2003) (Sculier, 2001). La prise en charge hémodynamique est aussi primordiale aux urgences. Les signes révélateurs d'une tumeur trophoblastique gestationnelle sont communément des métrorragies persistantes inexpliquées à distance d'un avortement spontané ou après une Interruption volontaire de la grossesse (environ $30 \%$ des cas) ou un accouchement (Golfier,2010) (Sierra Buerga, 2008) (El Fekih, 2010). Cette patiente avait une métrorragie minime persistante pendant quatre mois après une aspiration utérine. L'étude de Sierra-Bergua et al a relevé qu'une métrorragie intermittente était observée en post-partum pendant les huit mois, pourtant le diagnostic de choriocarcinome n'a été posé qu'après douze mois (Sierra Buerga, 2008). La présence d'une anémie sévère associée à un épanchement intra-péritonéal chez cette patiente devrait faire rechercher un hémopéritoine par érosion vasculaire utérine lors d'une atteinte de la séreuse (Golfier, 2010). La méconnaissance du diagnostic initial, l'insuffisance d'information et de suivi de la patiente expliquent l'évolution défavorable de sa maladie. Dans ce contexte défavorisé, il est parfois difficile de réaliser un dosage quantitatif d'hCG totale serique (hCG dimerique + chaines $\beta$ libres 1 ), toutefois un dosage hebdomadaire est nécessaire jusqu'à négativation confirmée par trois dosages successifs puis mensuels de 6 mois (Golfier, 2010). D’après une étude à Madagascar, seules 54,34\% ont pu se payer la surveillance du ßéta-hCG total sérique dont le coût équivaut à une semaine de revenu pour les patientes (Rajaonarison, 2015). La difficulté de prise en charge 
d'une môle hydatiforme en milieu rural a été décrite par des auteurs en Afrique. D'une part la situation d'indigence de la patiente aux revenus précaires, d'autre part la limitation des moyens techniques requis dans les centres de santé de périphérie pourraient expliquer la mauvaise observance au traitement (Fouogue Tsuala, 2011).

Le choriocarinome est une tumeur agressive mais très chimiosensible. A ce stade, une polychimiothérapie est recommandée. Il a été noté 70 à $78 \%$ de rémission après polychimiothérapie chez les patientes classée à haut risque (Golfier, 2010) (Chen, 2017).

Cette femme est sortie contre avis médical pour des raisons financières. Le traitement bien que cent fois plus coûteux que pour une tumeur à bas risque était totalement à sa charge.

\section{Conclusion}

Le succès du traitement du choriocarcinome gestationnel a été bien documenté, pourtant vue au stade de métastases avec des signes de détresses vitales, la patiente devrait être prise en charge dans une structure d'urgence. L'information et la sensibilisation de tout praticien médecin et sage-femme exerçant notamment en milieu rural sur ce type de pathologie pourrait éviter la survenue de complications fatales nécessitant des soins onéreux dans un pays à faible ressources.

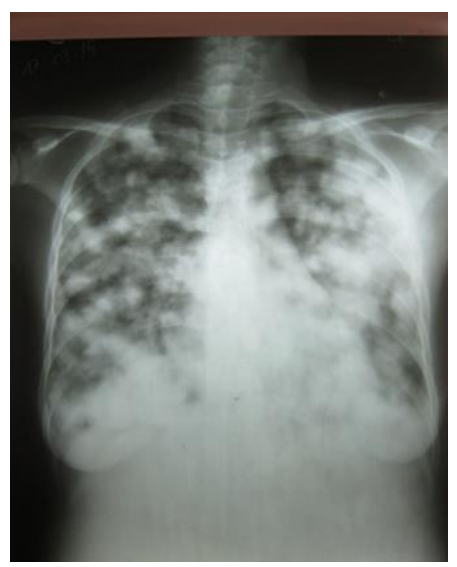

Fig. 1: Radiographie thoracique: Nodules parenchymateuses multiples 


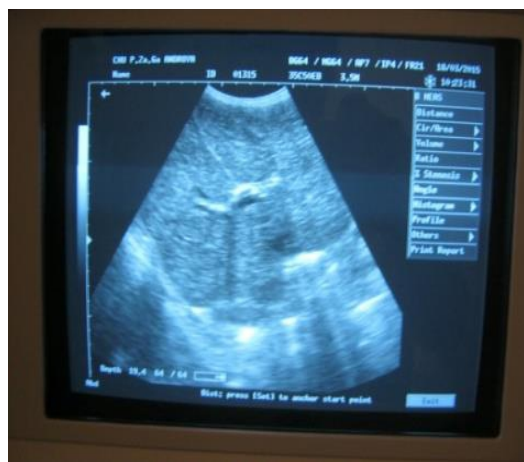

Fig. 2: Echographie abdominale : nodules hépatiques hyperéchogènes, contours bosselés en faveur d'une métastase.

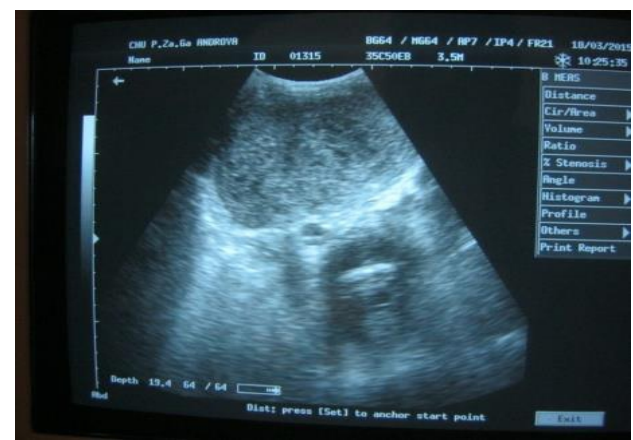

Fig. 3: Echographie pelvienne (coupe transversale) : utérus non gravide, masse hétérogène en nids d'abeille mesurant $63,8 \times 57,2 \mathrm{~mm}$.

\section{References:}

1. Golfier F, Massardier J, Guastalla JP, Trillet-Lenoir V, Frappart 1, Mathian B, ,Hajri T, Schott AM, Raudrant D. Prise en charge des maladies trophoblastiques gestationnelles. Journal de Gynécologie Obstétrique et Biologie de la Reproduction.2010;39S :F25-F35.

2. Rajaonarison JJC, Rakotondraisoa JM, Andrianampy HA, Randriambelomanana JA, Andrianampanalinarivo HR. Prise en charge des môles hydatiformes à l'Hôpital Universitaire Gynécologie Obstétrique de Befelatanana, Antananarivo. Rev méd Madag. 2015;5(1):510-5.

3. Mirambo M, Mazigo D, Jaka M, Kabangila R, Kombo H, Mshana SE, Faustine L, SENI J, Zinga MM. Case report: unsuspected uterine choriocarcinoma with lung metastasis. J Rural Trop Public Health 2010; 9:12-3.

4. Olezac AS, Papanicolaou I, Bengrine Lefevre L, Chouaid C. Choriocarcinome avec atteinte pulmonaire: stratégie diagnostique et thérapeutique. Rev Mal Respir 2009;26:769-72. 
5. 5-Sierra-Bergua B, Sánchez-Marteles M, Cabrerizo-García JL, Sanjoaquin-Conde I. Choriocarcinoma with pulmonary and cerebral metastases. Singapore Med J 2008;49(10):286-8

6. Leila El Fekih, Hela Hassene, Soraya Fenniche, Hajer Ben Abdelghaffar, Dalenda Belhabib, Mohamed Lamine Megdiche. Métastases pulmonaires révélatrices d'un choriocarcinome. La tunisie Medicale 2010; 88(1):49-51.

7. Sandeep Mehrishi, Aamir Awan, Anshu Mehrishi, Alan fein. Pulmonary tumor microembolism. Hospital Physician. 2004: 23-30

8. Yuanqian Chen, Linan Liu, Wei Zheng, Xin Zhang. Case Report Successful treatment of choriocarcinoma with multiple organ metastases after term delivery: a case report. Int J Clin Exp Med 2017;10(3):5468-5474

9. Cuvelier A, Muir JF. Détresse respiratoire hypoxémique: la ventilation non invasive protège les patients fragiles. Rev Mal Respir $2003 ; 20: 33-6$

10. Sculier JP, Berghmans T, Lemaitre F, Vallot F. La ventilation artificielle chez les patients atteints de cancer. Rev Mal Respir $2001 ; 18: 137-54$.

11. Fouogue Tsuala J, Fouélifack Ymélé F, Sando Z, Mboudou ET, Doh AS. Grossesse Môlaire et Difficultés de Prise en Charge en Milieu Rural : Illustration d'un Cas Clinique à l'Hôpital de District de Bogo (Extrême-Nord Cameroun). Clinics in Mother and Child Health. 2011(8):1-4. 